\title{
Uniportal video-assisted thoracoscopic sleeve resection
}

\author{
Dmitrii Sekhniaidze ${ }^{1}$, Diego Gonzalez-Rivas ${ }^{2,3}$ \\ ${ }^{1}$ Tyumen Regional Cancer Center, Tyumen, Russia; ${ }^{2}$ Department of Thoracic Surgery, Coruña University Hospital, Coruña, Spain; ${ }^{3}$ Minimally \\ Invasive Thoracic Surgery Unit (UCTMI), Coruña, Spain \\ Correspondence to: Dmitrii Sekhniaidze. Department of Thoracic surgery, Tyumen Regional Cancer Center, Tyumen, Russia. Email: skirrr@mail.ru.
}

Submitted Sep 19, 2015. Accepted for publication Jan 21, 2016.

doi: 10.21037/acs.2016.02.01

View this article at: http://dx.doi.org/10.21037/acs.2016.02.01

With continually growing interest in video-assisted thoracoscopic surgery (VATS) sleeve procedures in the international community, mastery of uniportal VATS sleeve resections is still a complex issue for surgeons. Several articles published recently have shown that VATS sleeve procedures can be performed safely (1-3). In this article the technique of a complex uniportal VATS sleeve resection is presented.

\section{Case 1}

\section{Clinical vignette}

The patient is a 67 year-old woman with a left upper lobe squamous cell carcinoma (cT3N0M0) involving mediastinal pleura and the pulmonary artery. Her pulmonary function testing indicated $43 \%$ of her predicted forced expiratory volume in one second (FEV1). Uniportal VATS double sleeve left upper lobectomy was scheduled.

\section{Surgical technique}

The patient was set in the lateral position. After singlelung ventilation, a $5 \mathrm{~cm}$ long incision was made in the fifth intercostal space (Video 1). The first step was revision and dissection of the mediastinal pleura, division of the pulmonary ligament, dissection of lymph nodes from stations 5, 6, 8 and 9 and visualization of the lower lobe vein (Video 2). The extra-pericardial part of the upper lobe vein was involved in the tumor. Therefore the pericardium was opened and both the upper lobe vein and left pulmonary artery were dissected. The upper lobe vein was divided by an endostapler. Then, the left main bronchus and lower lobe bronchus were dissected and cleared. After that, the ductus arteriosus was dissected and divided. The fissure was completed by endostaplers. After dissection of the lower lobe artery and division of the lingular artery, the possibility of resection was confirmed. Before clamping the left pulmonary artery, 5,000 units of heparin were injected intravenously. The left pulmonary artery and lower lobe artery were occluded by placing an arterial clamp and bulldog-clamp respectively. The arterial clamp was placed in the anterior portion of the incision. After that, the upper sleeve lobectomy with sleeve pulmonary artery resection en-bloc was performed. The specimen was temporarily placed in the diaphragmatic sinus. Then, after lymph node dissection from station 7, we performed bronchial and arterial anastomoses. The bronchial anastomosis was performed with a continuous suture (V-loc 3-0). The water probe was negative. The arterial anastomosis was performed with a continuous suture (prolene 4-0) and covered by oxidized regenerated cellulose (Surgicel). The specimen was removed by endo-catch. Surgery time was 300 minutes. Blood loss was $200 \mathrm{~mL}$.

\section{Case 2}

\section{Clinical vignette}

The patient is a 54 year-old man with a right upper lobe squamous cell carcinoma (cT4N0M0) involving the right main bronchus and superior vena cava. After three courses of neoadjuvant chemotherapy (gemcitabine and cisplatin), the tumor size was reduced to some extent. Pulmonary function testing showed $74 \%$ of his predicted FEV1. Uniportal VATS total sleeve carinal pneumonectomy with vena cava resection was scheduled.

\section{Surgical technique}

The patient was set in the lateral position. After singlelung ventilation, a $5 \mathrm{~cm}$ long incision was made in the fifth 
intercostal space. The first step was revision, dissection and partial resection of the superior vena cava by endostapler. The next step was dissection of lymph nodes from stations 2, 3 and 4 and visualization of the anterior walls of the trachea and carina. Then, the upper lobe vein was dissected. The posterior part of the azygos vein was dissected, clipped and divided. Following dissection of the posterior wall of the carina, the possibility of resection was confirmed. The next step was dissection and consistent division of the inferior pulmonary vein, superior pulmonary vein and pulmonary artery by endostaplers. After mobilization of the right lung and carina with lymph node dissection from station 7 , total carinal resection was performed with a double lumen tube. Frozen section histological examination of the tracheal margin was positive therefore we resected one tracheal ring. When the specimen was removed and hemostasis was checked, we commenced high frequency ventilation. The tracheabronchial anastomosis was performed with a continuous suture. We used the V-Loc 3-0 wound closure device, which facilitates secure anastomosis closure without knot-tying. The water probe was negative. To cover the anastomosis, we used a mediastinal pleural flap. The operation time was 280 minutes. Blood loss was $100 \mathrm{~mL}$. Flexible bronchoscopy at the end of the procedure showed a normal endobronchial view of the anastomosis.

\section{Comments}

\section{Clinical results}

There were no postoperative complications. Final pathology revealed pT3N0M0 in case 1 and. pT4N0M0 in case 2 .

\section{Advantages}

The uniportal approach for VATS sleeve procedures provides a perfect direct view, which is required to perform reliable bronchial and vascular anastomoses. In the case of conversion, the uniportal approach can be expanded to thoracotomy within 1 or 2 minutes. Another advantage is that the instruments are inserted parallel to the thoracoscopy. This makes the technique of performing arterial anastomosis similar to open surgery.

\section{Caveats}

Usually, the intrapericardial part of the upper lobe vein is involved in the tumor in double sleeve cases. It is impossible to execute the uniportal VATS principle of 'first artery then vein' in double sleeve cases. This is the serious obstacle to division of the upper lobe vein by endostaplers by the uniportal approach. To overcome this situation, we have to open the pericardium in order to lengthen the vessel. We can use a $30 \mathrm{~mm}$ vascular stapler for open surgery or ligature and clips to divide the vein safely. To evaluate tumor invasion in the lower lobe artery, the fissure should be divided. This is the main point to confirm the possibility of the double sleeve procedure. As a general rule, the left pulmonary artery is very short. Therefore the ductus arteriosus must be dissected and divided. It provides $1-1.5 \mathrm{~cm}$ of length to the vessel and this is very important for placing an arterial clamp safely. After lung mobilization, carinal resection by the uniportal approach must be performed with a double lumen tube to avoid any blood aspiration into the left main bronchus. After removing the specimen and ensuring hemostasis, it is necessary to start high frequency ventilation in order to perform the anastomosis more easily.

\section{Acknowledgements}

None.

\section{Footnote}

Conflicts of Interest: The authors have no conflicts of interest to declare.

\section{References}

1. Xu X, Chen $\mathrm{H}$, Yin $\mathrm{W}$, et al. Thoracoscopic half carina resection and bronchial sleeve resection for central lung cancer. Surg Innov 2014;21:481-6.

2. Gonzalez-Rivas D, Delgado M, Fieira E, et al. Double sleeve uniportal video-assisted thoracoscopic lobectomy for nonsmall cell lung cancer. Ann Cardiothorac Surg 2014;3:E2.

3. Huang J, Li J, Qiu Y, et al. Thoracoscopic double sleeve lobectomy in 13 patients: a series report from multicenters. J Thorac Dis 2015;7:834-42.

Cite this article as: Sekhniaidze D, Gonzalez-Rivas D. Uniportal video-assisted thoracoscopic sleeve resection. Ann Cardiothorac Surg 2016;5(2):145-146. doi: 10.21037/ acs.2016.02.01 